\title{
Correlative Spectromicroscopy Software Development on the SGM and Mid-IR Beamlines at the CLS
}

$\underline{\text { James J. Dynes }}{ }^{1, *}, Z^{2}$ achary Arthur ${ }^{1}$, Stuart Read $^{1}$, Jarvis Stobbs $^{1}$, Tom Z. Regier $^{1}$, Scott M. Rosendahl $^{1}$

1. Canadian Light Source, Science, Saskatoon

* Corresponding author, email_james.dynes@lightsource.ca

To fully understand the chemical spatial nature of your samples often requires that you use more than one type of spectromicroscopy and that you correlate the results. The new soft X-ray microprobe at the SGM beamline uses a Kirkpatrick Baez (KB) mirror system to achieve a beam spot size of $10 \mathrm{X} 10 \mu \mathrm{m}^{2}$, similar to the spot size obtained by current Fourier transform infrared (FTIR) microscopes. Correlative spectromicroscopy using soft X-rays $(250$ to $2200 \mathrm{eV})$ and infrared radiation $(0.1$ to $0.5 \mathrm{eV})$ now becomes very attractive for mapping the elemental and chemical species for materials with structures several microns in size. Here we report on our progress to develop software for image analysis of the microprobe data, with the aim to correlate the elemental and chemical species in a sample using data collected from the SGM microprobe and an FTIR microscope.

To develop our correlative capabilities between the SGM and Mid-IR beamlines we used a model organic mixture where selected materials were pressed into a $\mathrm{KBr}$ disk. The compounds included graphite (black), citric acid (white), $\mathrm{CuCO}_{3}$ (blue) and $\mathrm{K}_{3} \mathrm{Fe}(\mathrm{CN})_{6}$ (orange) (Figure 1). Besides their color, the compounds were selected based on their different organic functional groups. The $\mathrm{KBr}$ disk was fastened to a brass plate, which could be mounted on the sample holders from both microscopes.

The SGM XAS endstation has a hexapod sample positioner/holder and fluorescence detector array consisting of four silicon drift detectors positioned at $30 \mathrm{~mm}$ from the sample with specific geometry, providing multiple perspectives of the sample. The data is collected using SPEC from Certified Scientific Software and stored in the hdf5 format. The partial fluorescence yield (pfy) at the C K-edge was obtained from excitation emission matrix [1]. We are currently developing an in-house python-based program for image analysis.

The Mid-IR microscope used for the study was an Agilent Cary 670 FTIR Spectrometer with a Cary 620 Microscope (128x128 pixel Focal Plane Array detector). The light source was a globar. Reflectance spectra were measured and the absorption spectra were calculated using the Kramers-Kronig transformation. Data was analyzed using open-source spectral data processing and analysis software tools building on the Orange multivariate data analysis, visualization and mining suite (https://orange.biolab.si/) [2]. This software is developed by the CLS Mid-IR beamline in collaboration with infrared beamlines at SOLEIL and Elettra and is available under an open-source license, enabling easy collaboration, contribution of new features and verification of processing algorithms.

Soft X-ray spectromicrocopy. We collected 33 images from a 6 x $6 \mathrm{~mm}$ area on the $\mathrm{KBr}$ disk from 280 to $320 \mathrm{eV}$. Each image consisted of over 14000 data points which were interpolated at a spatial resolution of $10 \mu \mathrm{m}$. 
Infrared Spectroscopy. We collected 256 co-added interferograms from an $1.4 \mathrm{x} 2.1 \mathrm{~mm}$ area consisting of six $704 \mu \mathrm{m}^{2}$ tiles ( $2 \times 3$ tile grid) with a pixel size of about $6 \mu \mathrm{m}$. Each of the resulting 98304 spectra covered a $900-3950 \mathrm{~cm}^{-1}$ energy range with $4 \mathrm{~cm}^{-1}$ resolution.

The brass plate with the attached sample was easily mounted on both microscope's sample holder. We were able to develop python-based software that made an image sequence from the 33 soft X-ray images and extract spectra from selected regions (Figure 2). Also, individual images could be saved as Tiff images to be used in ImageJ for correlative analysis.

We erred when we made the model organic mixture, as IR spectra could only be collected from the citric acid and $\mathrm{Cu}$ carbonate as the other compounds absorbed all the infrared radiation. In the future we will make a sample such that we can collect the IR spectra using transmittance.

Future developments of the soft X-ray software will include spectral fitting of the image sequence using reference spectra. The soft X-ray data is slightly distorted as the sample is not perpendicular to the beam, thus spectral distortion correction will be required before it can be accurately correlated with the IR data. We expect to have these developments complete by the time of the XRM2018 conference.

\section{References}

[1] A.W. Gillespie et al., in “Advances in Agronomy” ed. D.L. Sparks, (Academic Press, NY), p. 1

[2] M. Toplak et al, Synchrotron Radiation News 30 (2017) 40.

[3] The authors acknowledge support from the Canadian Light Source.
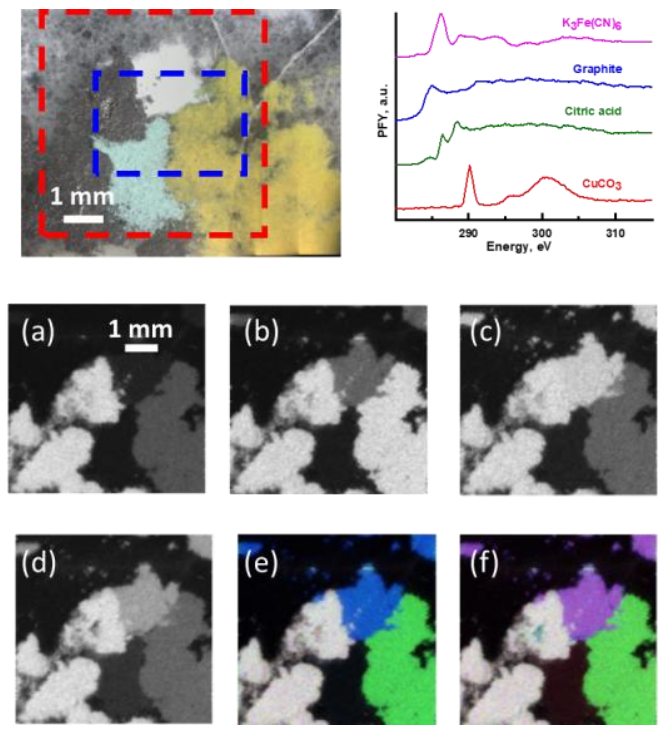

Figure 1. Optical image and C K-edge spectra and images at selected energies (a) 285 , (b) 286.3 , (c) 288.6 , (d) $290.3 \mathrm{eV}$ and overlay of images (e) $285,286.3$, $288.6 \mathrm{eV}$, and (f) $290.3,286.3,288.6 \mathrm{eV}$. Red square (large) $=$ Soft X-ray and Blue square (inner) $=$ IR area of study.
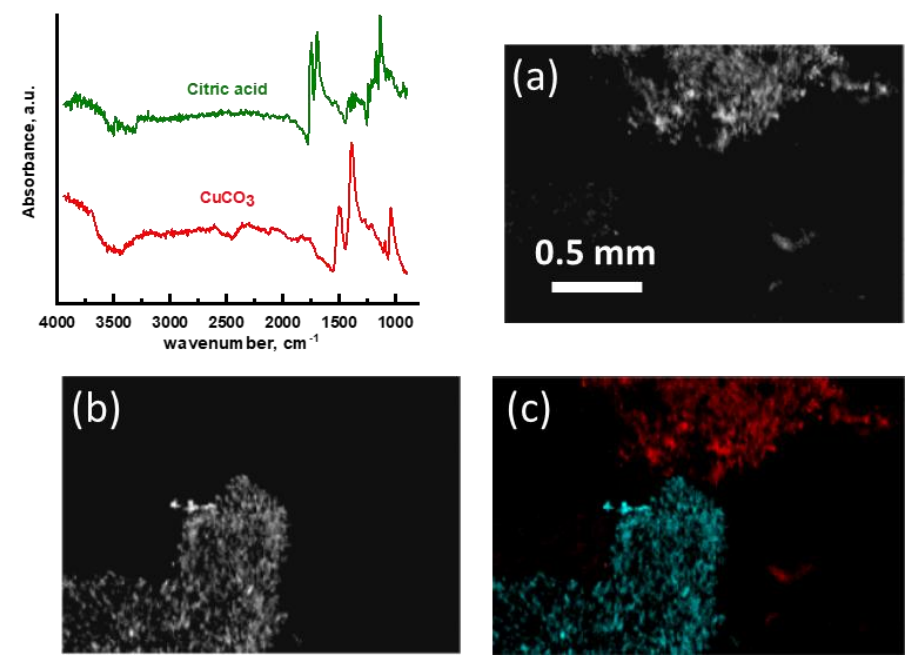

Figure 2. IR spectra and images. Images where derived by integration of the peaks for (a) citric acid, $1165-1185 \mathrm{~cm}^{-1}$ and (b) carbonate, $1438-1543 \mathrm{~cm}^{-1}$. (c) Overlay of citric acid and carbonate images. 